\section{Eliminationsdiät und Stillen: Gefahr für die Knochendichte?}

\author{
Eine Forschergruppe aus Tampere in Finnland wollte wissen, ob \\ Mütter atopischer Kinder bedenkenlos länger stillen können und ob \\ sich eine Eliminationsdiät auf die Knochendichte auswirkt.
}

$\mathrm{D}$ azu wurden 24 Mütter untersucht, bei deren Kindern sich im Laufe der ersten sechs Lebensmonate ein atopisches Ekzem entwickelt hatte (Fallgruppe), sowie 25 Mütter von Kindern ohne Ekzem (Kontrollgruppe). 19 der Mütter von Ekzemkindern hielten sich vorübergehend an eine Diät ohne Fisch, Eier oder Erdnüsse. Manche verzichteten zusätzlich auf

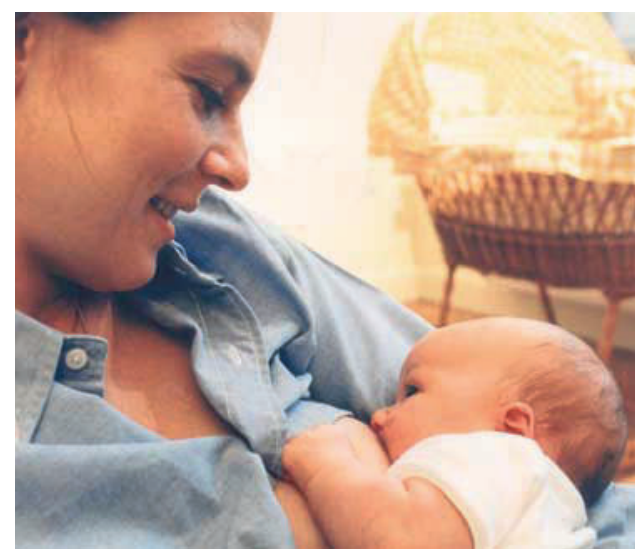

Beeinträchtigt das Stillen die Knochendichte der Mutter?

Kuhmilch und eventuell auch Fleisch. Zum Zeitpunkt der zweiten Regelblutung nach der Geburt erfassten die Autoren die Knochendichte (LWS, rechter Oberschenkelhals, distaler Radius) und Omega-3-/Omega-6-Fettsäurespiegel im venösen Blut.

Die Mütter der atopischen Kinder stillten durchschnittlich länger ohne Beikost als Mütter der Kontrollgruppe (4,1 vs. 2,8 Monate). In der Fallgruppe zeigte sich ein Trend zu einer um 4 bis $6 \%$ niedrigeren Knochendichte. Dieser Unterschied erklärte sich jedoch hauptsächlich durch das unterschiedliche Körpergewicht. Die Konzentration der Omega-3-Fettsäuren im Blut der Mütter war in Fall- und Kontrollgruppe vergleichbar; bei Müttern atopischer Kinder waren Linolensäure niedriger und $\alpha$-Linolensäure höher konzentriert (beides Omega-6-Fettsäuren) als im Blut der Kontrollgruppe. Diese Differenzen beruhten auf unterschiedlichem Fischkonsum, Dauer der Amenorrhoe und Gesamtstillperiode.
K inder, die auf einem Bauernhof groß werden, bekommen im Laufe ihres Lebens seltener allergische Erkrankungen. Kliniker in ländlichen Gebieten von Österreich, Deutschland und der Schweiz wollten wissen, in welchem Alter der Kontakt zu Schweinen, Kühen und Pferden erfolgen muss.

Gemäß einem standardisierten Fragebogen gaben Eltern von insgesamt 2618 Kindern Auskunft über Atemwegsbzw. allergische Erkrankungen ihrer Sprösslinge sowie über die Lebensgewohnheiten auf ihrem Bauernhof. Zusätzlich wurden bei 901 Kindern aus Bauern- und Nichtbauernfamilien Tests auf spezifische Serum-IgE-Antikörper (u.a. gegen Kuhepithelien, Hausstaubmilben sowie andere Inhalations- oder Nahrungsallergene) durchgeführt.

Auf dem Bauernhof aufgewachsene Kinder bekamen signifikant seltener allergische Erkrankungen wie Asthma oder Heufieber. Am wirksamsten geschützt waren Kinder, die bereits im ersten Lebensjahr dem Kontakt mit Stalltieren ausgesetzt waren und zusätzlich rohe Kuhmilch bekommen hatten; nicht ganz so wirksam war eine Exposition erst im

\section{Fazit}

Weder das Stillen noch eine vorübergehende Eliminationsdiät beeinflussten die Knochendichte der Mütter. Falls eine solche Diät sinnvoll erscheint, kann sie für einen begrenzten Zeitraum bedenkenlos empfohlen werden. Allerdings sollten Mütter mit niedrigem BMI besonders darauf achten, dass sie alle notwendigen Nährstoffe zu sich nehmen. Dr. I.E. Wagener, Berlin

Holmberg-Marttila D et al. Do combined diet and prolonged breastfeeding of an atopic infant jeopardise maternal bone health? Clin Exp Allergy 2001; 31: 88-94

\title{
Kindheit beim Bauern schützt vor Asthma
}

\author{
Wer im ersten Lebensjahr Kontakt mit Stalltieren hatte, darf sich \\ glücklich schätzen: Das Risiko, an Rhinitis allergica oder Asthma zu \\ erkranken, ist deutlich niedriger.
}

zweiten bis fünften Lebensjahr. Erfolgte der Kontakt zu Stalltieren besonders häufig, länger als 20 Minuten täglich sowie kontinuierlich bis zum fünften Lebensjahr, war die Rate allergischer Erkrankungen am niedrigsten. Aber auch bereits die pränatale Exposition konnte das Allergierisiko mindern: Kinder von Müttern, die während der Schwangerschaft auf dem Bauernhof gearbeitet hatten, waren besser geschützt.

\section{Fazit}

Die Ursache für diese Assoziation ist unbekannt. Eine Hypothese lautet: Auf Bauernhöfen finden sich höhere Konzentrationen von Endotoxinen und anderen mikrobiellen Faktoren. Inhaliert oder geschluckt könnten diese Faktoren z.B. über die Bildung von bestimmten Interleukinen einen Anstieg von $\mathrm{T}_{1}$ - $\mathrm{Hel}$ ferzellen und damit eine veränderte Immunantwort bewirken - das Risiko allergischer Reaktion sinkt.

$D D$

Riedler J et al. Exposure to farming in early life and development of asthma and allergy: a cross-sectional survey. Lancet 2001; 358: 1129-33 